\title{
The Problem of Freezing of Copper Water Heat Pipes
}

\author{
Subas Acharya ${ }^{1}$, Valeria Barra ${ }^{2}$, Dean Duffy ${ }^{3}$, \\ David A. Edwards ${ }^{4} \dagger$, and VRushaly Singlot ${ }^{1}$ \\ ${ }^{1}$ University of Texas, Dallas, USA \\ ${ }^{2}$ University of Colorado, Boulder, USA \\ ${ }^{3}$ NASA, USA \\ ${ }^{4}$ University of Delaware, USA
}

(Communicated to MIIR on 11 September 2021)

Study Group: 34th MPI Workshop, 25-29 June 2018, Claremont Graduate University

Communicated by: Hilary Ockendon

Industrial Partner: Jet Propulsion Laboratory.

Presenter: Juan Cepeda-Rizo

Team Members: Subas Acharya, University of Texas, Dallas; Valeria Barra, University of Colorado, Boulder; Dean Duffy, NASA; David A. Edwards, University of Delaware; Vrushaly Singlot, University of Texas, Dallas

Industrial Sector: Aerospace

Key Words: Freezing, Heat transfer, Porous medium, Stefan problem

MSC2020 Codes: 80

\section{Summary}

To cool electronic components of spacecraft, engineers are considering the use of heat pipes, which are sealed copper pipes containing an annular copper wick saturated with water. Water vapor from the hot end travels to the cold end, where it condenses in the wick and flows back to the hot end.

In space, the cold end can freeze the water inside, which can cause two modes of pipe failure. First, if water accumulates in the cold end, then freezes, it can burst the pipe wall. Second, with the repeated expansion of the freezing water, pores in the copper wick can enlarge, reducing the speed of transport and hence the heat pipe's effectiveness.

In this work we will examine both types of failures, with an eye toward establishing tolerances below which we expect the wick to remain functional.

$\dagger$ Corresponding author: dedwards@udel.edu 


\section{Section 1: Introduction}

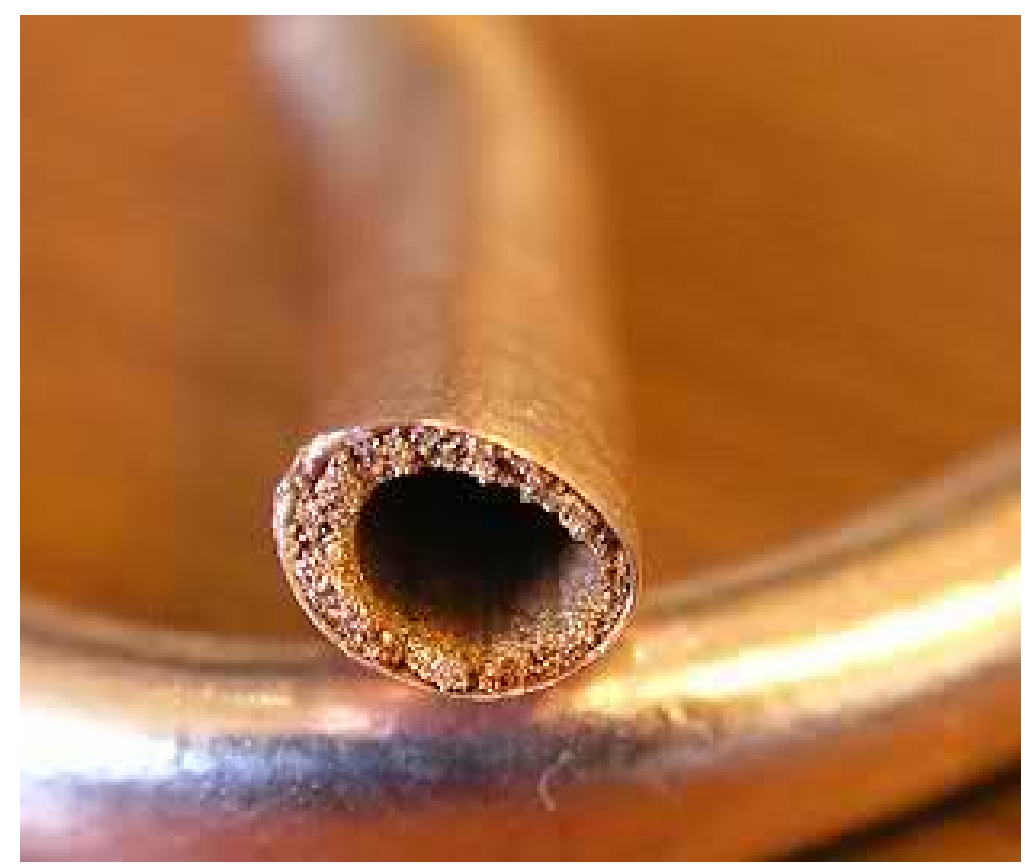

Figure 1.1. Typical heat pipe with sintered wick [2].

It is imperative to cool electronic components of spacecraft in order to ensure their continued performance. One possibility for cooling such components is the use of heat pipes, which use the heat capacity of a working fluid to dissipate heat. In the case we analyze, these devices consist of a copper pipe (sealed at both ends) with an annular sintered copper wick inside (see Fig. 1.1), and contain water as the working fluid. Capillarity forces induced by the porosity of the wick enhance the circulation of water in the pipe. The core of the pipe is then set to near-vacuum.

Figure 1.2 shows a schematic. Liquid water at the hot end is vaporized, absorbing heat. Given that the pipe is sealed, the water vapor travels to the other (cold) end, where it condenses in the wick, which is adjacent to the cold environment of space. The water then travels through the wick to the hot end, where the process repeats.

These designs have been used extensively in terrestrial applications (cooling computer chips, etc.), but in those cases the cold end has not been held at temperatures which would freeze the water inside. Therefore, care must be taken to ensure that the pipe will not fail in such an environment. To do that, experimentalists run a series of freeze-thaw cycles to determine whether the repeated freezing and thawing of the water inside will damage the copper compoenents.

There are two modes of failure which we wish to investigate. In the first, water accumulates in the cold end, then freezes. The resulting expansion of the liquid water is enough to burst the pipe wall, and has been seen experimentally [3]. We call this wall failure. 


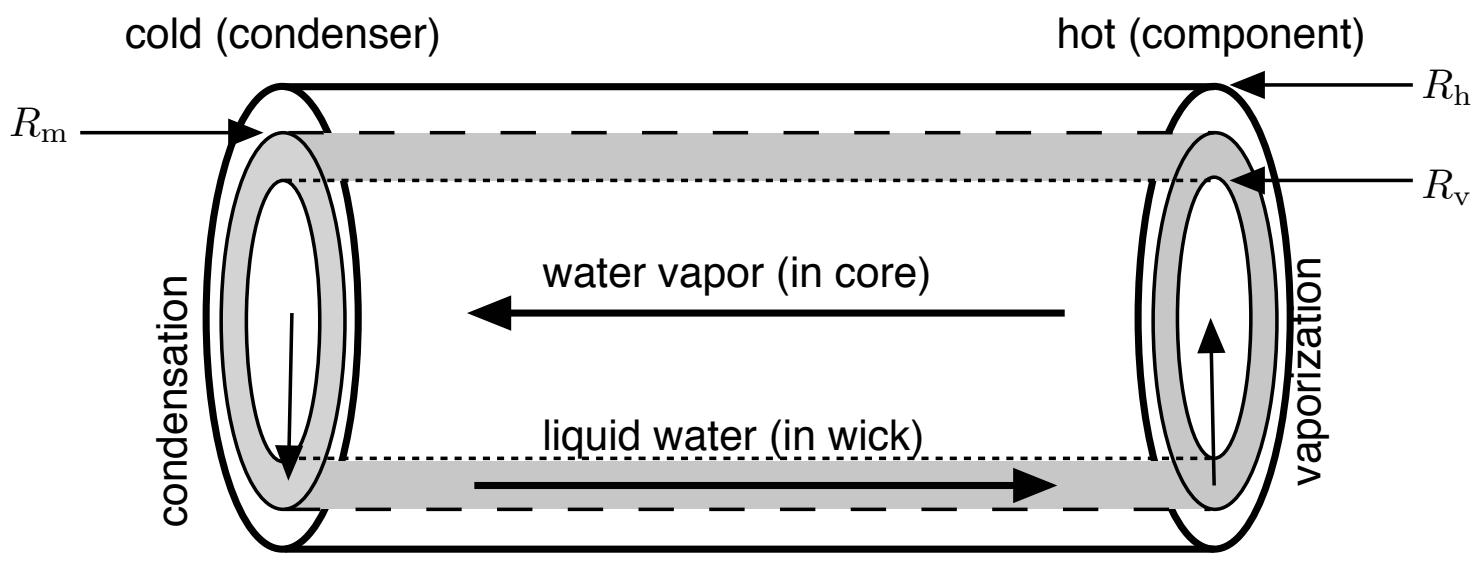

Figure 1.2. Schematic of heat pipe. Grey region is wick; region outside is pipe wall, region inside is evacuated core.

The sintered copper wick forms a porous medium with a small pore size, which enhances liquid transport from the cold end to the hot end. In the second type of failure, water in the wick freezes and expands, deforming the porous media structure. With a larger pore size, the speed of transport in the wick decreases, reducing the heat pipe's effectiveness. We call this wick failure.

In this work we will examine both types of failures, with an eye toward establishing tolerances below which we expect the wick to remain functional. 


\section{Section 2: Pipe Failure}

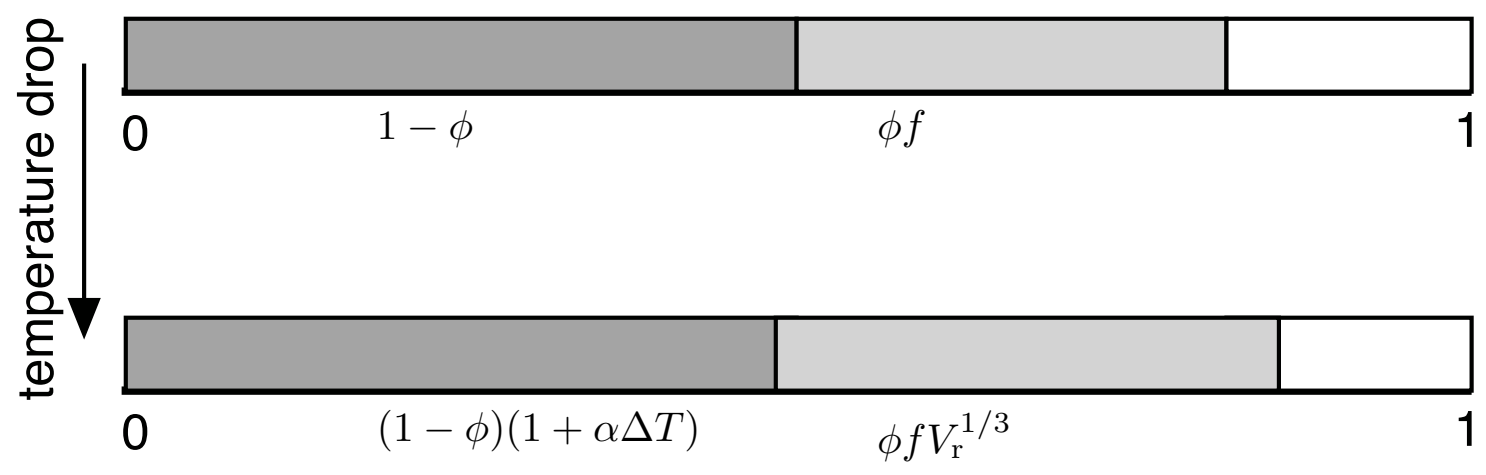

Figure 2.1. One-dimensional model of expansion. Top diagram: $T_{\mathrm{w} 0}=20^{\circ}$ C. Lower diagram: $T_{\mathrm{i} 0}=0^{\circ} \mathrm{C}$. Dark grey: copper. Light grey: water. White: void space.

We begin by examining the case of pipe failure. We introduce a simple one-dimensional model as shown in Fig. 2.1. We scale the width of the wick to be 1, and just consider the proportion of each of the three components in the cross section.

At roughly room temperature $\left(T_{\mathrm{w} 0}=20^{\circ} \mathrm{C}\right.$, where the subscript "w" refers to "water"), the void fraction of the porous medium is given by $\phi(0 \leq \phi \leq 1)$. Hence the proportion of copper in the wick is given by $1-\phi$. Then the voids are filled with water to some percentage $f$ (called the charging factor), so the fraction of water in the cross-section is given by $\phi f$.

Next, we bring the temperature of the pipe down to $T_{\mathrm{i} 0}=0^{\circ} \mathrm{C}$, where the subscript "i" refers to "ice". We choose this value since this is the where the density of water is lowest, which corresponds to the largest expansion. The copper will contract by a factor $1+\alpha \Delta T$, where $\alpha$ is the linear thermal expansion coefficient, and $\Delta T=T_{\mathrm{i} 0}-T_{\mathrm{w} 0} \cdot \alpha$ is roughly constant for a metal like copper. Note also that $\Delta T=-20^{\circ} \mathrm{C}<0$, so the metal does indeed contract.

On the other hand, the water will expand. The coefficient of thermal expansion for water is not constant, so we use specific values of the density $\rho$ of water. In particular, we have that the volume of water will expand by the following volumetric expansion factor:

$$
V_{\mathrm{r}}=\frac{\rho_{\mathrm{i}}}{\rho_{\mathrm{w}}} \text {. }
$$

We note that $V_{\mathrm{r}}>1$ since the density of water is higher at room temperature than at freezing. To convert this to a linear expansion factor, we simply take the cube root. Hence we have that at freezing, the amount of space taken up by the copper and the water is given by

$$
\underbrace{(1-\phi)(1+\alpha \Delta T)}_{\text {copper }}+\underbrace{\phi f V_{\mathrm{r}}^{1 / 3}}_{\text {water }} \text {. }
$$


We say that the pipe has failed if the above expression is greater than 1 . Hence the ice has expanded enough that it will burst the pipe wall, or that it will expand into the inner core. Once in the core, the water can pool during thaw cycles, eventually freezing and bursting the pipe during a later cycle.

Typically $\phi$ is determined for a particular wick, and $f$ is at the discretion of the manufacturer. Therefore, we construct an upper bound for $f$ given a particular $\phi$ :

$$
\begin{aligned}
(1-\phi)(1+\alpha \Delta T)+\phi f V_{\mathrm{r}}^{1 / 3} & \leq 1 \\
\phi f V_{\mathrm{r}}^{1 / 3} & \leq 1-(1-\phi)(1+\alpha \Delta T) \\
f & \leq \frac{-\alpha \Delta T+\phi(1+\alpha \Delta T)}{\phi V_{\mathrm{r}}^{1 / 3}} \\
& \leq \frac{1+\alpha \Delta T}{V_{\mathrm{r}}^{1 / 3}}-\frac{\alpha \Delta T}{\phi V_{\mathrm{r}}^{1 / 3}} .
\end{aligned}
$$

If $\phi=1$ and there is no copper, the maximum loading percentage is $V_{\mathrm{r}}^{-1 / 3}$. This corresponds to the percentage of water that will expand to fill the entire volume upon freezing. If $\phi=0$ and there is no water, there is no maximum loading percentage.

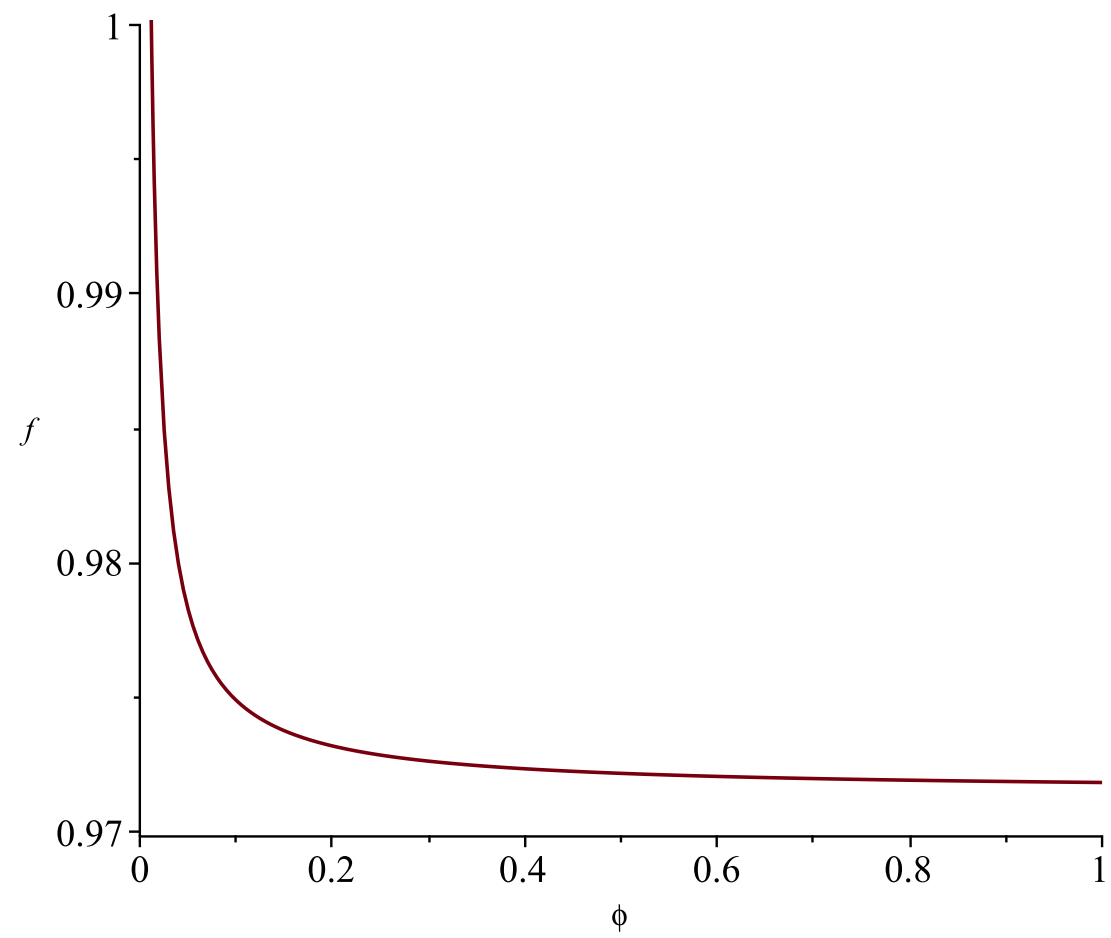

Figure 2.2. Plot of (2.2).

A graph of (2.2) for the values in Appendix A is given in Fig. 2.2; the upper limit for the charging factor is always over $97 \%$. How then to explain the failure in [3]? Recall that in terrestrial applications, freezing is not an issue, so the charging factor can be 1 , or even higher, if additional water is allowed to pool in the core. Hence a heat pipe designed for 
terrestrial uses could undergo pipe failure upon freezing. Fortunately, heat pipes designed for use in space are usually charged to only about $f=0.9$, which is well under the upper bound in (2.2).

We conclude by noting that the failure of the heat pipe is quite different from the failure of copper water pipes in houses.

In a house water pipe, the cold pipe freezes the water from the outside in, forming an ice annulus. As the annulus solidifies into a solid plug, the ice then begins to expand parallel to the pipe. Expansion toward the house increases pressure on the water in the pipe downstream of the plug. It is this pressure which then bursts the pipe. ${ }^{*}$ In contrast, the heat pipe is near vacuum. So the ice itself must burst the copper pipe.

Moreover, this phenomenon is quite different in terms of geometry. Most engineering discussions of pipe bursting focus on the case where the pipe wall thickness is small compared to the pipe diameter. But we see from Appendix A that the pipe wall thickness is comparable to the pipe diameter, which is a regime not generally discsussed.

* This is why house pipes leak water when burst. It is also why it is suggested to leave the faucet dripping when freezing is possible (as this relieves the pressure in the line). 


\section{Section 3: Wick Failure}

We next examine the case of wick failure. We wish to see if the thermal expansion of the water as it freezes will deform the structure of the pores in the wick. If the pores become too large, that may lessen the wicking effect and cause the heat pipe to fail. (To our knowledge, this has not been observed experimentally.)
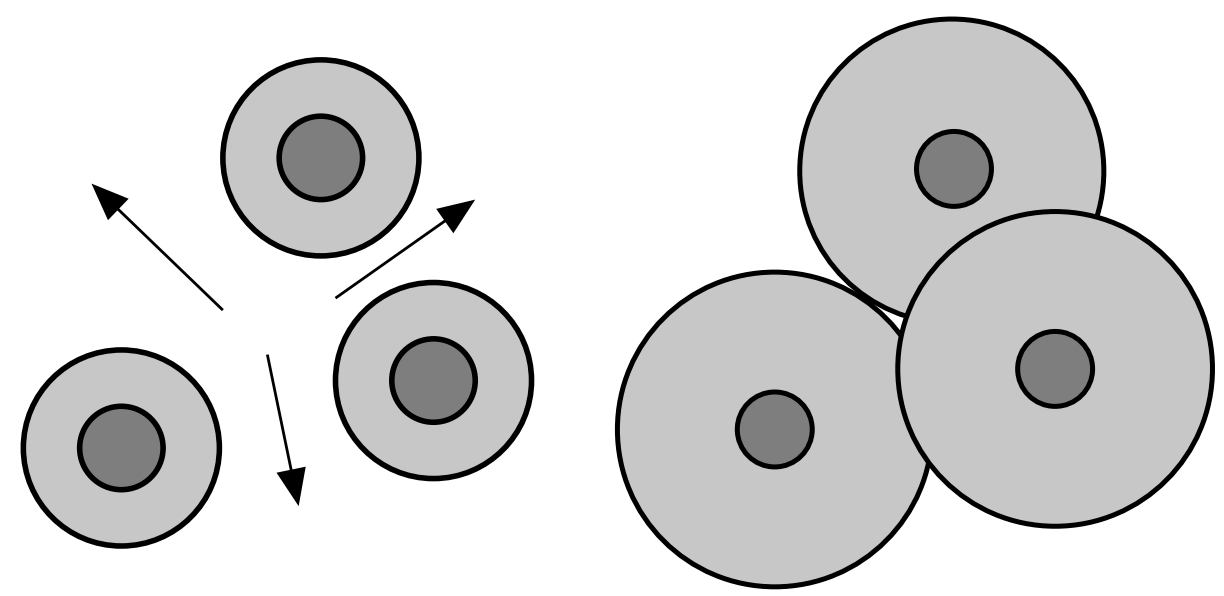

Figure 3.1. Schematic of pumping action caused by freezing. Dark grey circles correspond to copper; light grey circles correspond to ice. Left: illustration of pumping process at beginning of freezing. Water flows in direction of arrows. Right: As freezing continues, a small quantity of water is trapped by the overlapping ice circles.

As the porous media itself is made of copper, it will conduct heat quickly. Hence we expect the ice to move outward from the copper toward the center of the pores. In general, this will cause a pressure gradient which will pump water through the porous media (see left of Fig. 3.1). Since the inner core is near vacuum, water will tend to flow from the outer portions of the pipe to the empty inner portion of the wick. Then that water will also come into contact with the cold copper wick, where it will freeze.

However, as the ice from neighboring beads comes together, they can isolate a water pocket (small white area in center of right of Fig. 3.1). As this pocket freezes, it will exert pressure on the neighboring ice. It is our belief that if these pockets are small enough, they will not cause enough pressure to distort the wick matrix, which has of course been trapped in ice from the beginning.

We now present a toy problem to illustrate our analysis. But first, some caveats:

1. The idea of isolating the copper beads in the water is not realistic. We could think of them being connected by thin sinter connections made of copper, or consider the microstructure more carefully (see Section 4).

2. In the right of Fig. 3.1, the ice region is shown as a series of overlapping circles, and we will continue that model here. However, in reality surface energy considerations would cause the boundaries of the water pocket to be convex. 
3. In two dimensions, it is much easier to trap water than in three dimensions. In particular, consider the problem of circle packing in two dimensions. This will cause many inclusions, as described below. But the problem of sphere packing does not. In particular, a perfect sphere packing still has a continuous pore structure through it. Hence there will have to be more overlap (as in the right of Fig. 3.1) before the inclusions are closed. This will reduce their surface area.

In the light of the above, the analysis we present will give a conservative estimate of the volume of inclusions - most assuredly too conservative.
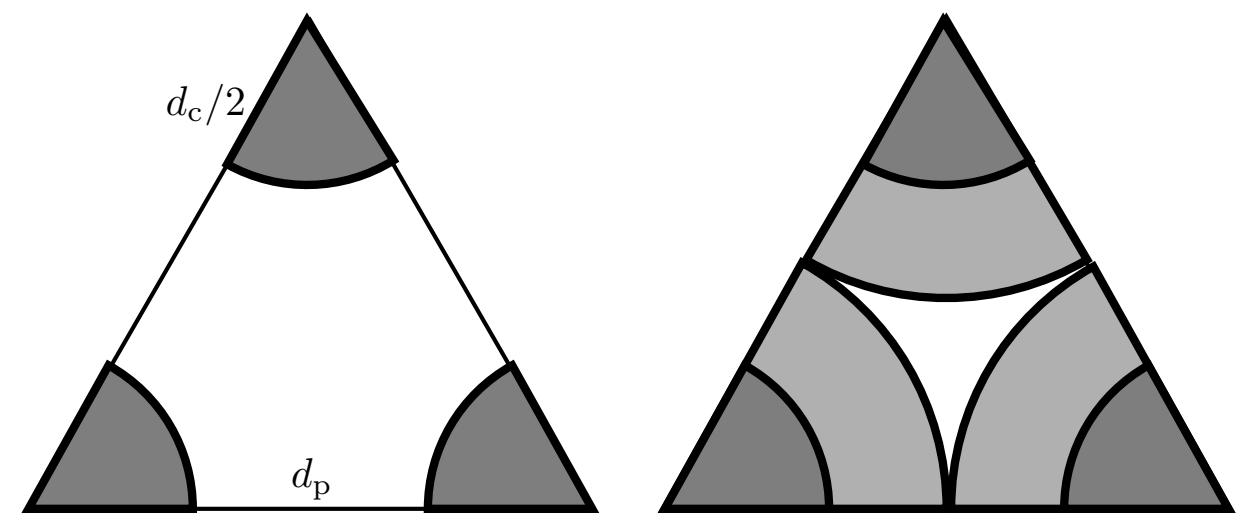

Figure 3.2. Schematic of trapping water by freezing of triangular lattice. Triangle is repeated in six-fold symmetry in lattice. Dark grey regions correspond to copper, light grey regions correspond to ice, white regions correspond to water. Left: array before freezing. Right: Trapping of water in matrix.

We begin by considering a triangle (really part of a hexagonal array), as shown in Fig. 3.2. The triangle is equilateral with side length $d_{\mathrm{p}}+d_{\mathrm{c}}$, where $d_{\mathrm{p}}$ is the diameter of the pore and $d_{\mathrm{c}}$ is the diameter of the bead (the subscript "c" stands for "copper"). Hence the area of the triangle is given by

$$
A_{\triangle}=\frac{\left(d_{\mathrm{p}}+d_{\mathrm{c}}\right)^{2} \sqrt{3}}{4} .
$$

Taken together, the sectors form a semicircle of diameter $d_{\mathrm{c}}$ with area

$$
A_{\mathrm{c}, \triangle}=\frac{\pi}{2}\left(\frac{d_{\mathrm{c}}}{2}\right)^{2}=\frac{\pi d_{\mathrm{c}}^{2}}{8}
$$

After freezing, the sectors form a semicircle of diameter $d_{\mathrm{c}}+d_{\mathrm{p}}$ with area

$$
A_{\mathrm{i}, \triangle}=\frac{\pi\left(d_{\mathrm{c}}+d_{\mathrm{p}}\right)^{2}}{8} .
$$

Thus the fraction $F$ of the water in the pore originally that becomes trapped is given by the following:

$$
F_{\triangle}=\frac{A_{\triangle}-A_{\mathrm{i}, \triangle}}{A_{\triangle}-A_{\mathrm{c}, \triangle}}=\frac{\left[\left(d_{\mathrm{p}}+d_{\mathrm{c}}\right)^{2} \sqrt{3} / 4\right]-\pi\left(d_{\mathrm{c}}+d_{\mathrm{p}}\right)^{2} / 8}{\left[\left(d_{\mathrm{p}}+d_{\mathrm{c}}\right)^{2} \sqrt{3} / 4\right]-\pi d_{\mathrm{c}}^{2} / 8}=\frac{\left(d_{\mathrm{p}}+d_{\mathrm{c}}\right)^{2}(2 \sqrt{3}-\pi)}{2\left(d_{\mathrm{p}}+d_{\mathrm{c}}\right)^{2} \sqrt{3}-\pi d_{\mathrm{c}}^{2}} .
$$


With the values of the parameters from Appendix A, we have

$$
F_{\triangle}=0.130 \text {. }
$$

Note that:

1. Because of the symmetry involved with the equilateral triangle, $F_{\Delta}$ as given by (3.5) is actually the largest percentage that can be trapped. Random placement greatly reduces the size of the inclusions (see right of Fig. 3.1).

2. The analysis can be continued by hand (at least) for isosceles triangles. However, the analysis is complicated, must be broken into separate cases, and is not particularly illustrative, as (3.5) provides the upper bound.

Nevertheless, the process may be handled numerically for all triangles using Mathematica (see Appendix B). It is possible that separate cases may be needed here as well, depending on the relative lengths of the triangles. Also, Mathematica should be able to do the same sorts of calculations in three dimensions, but that is beyond the scope of this manuscript.
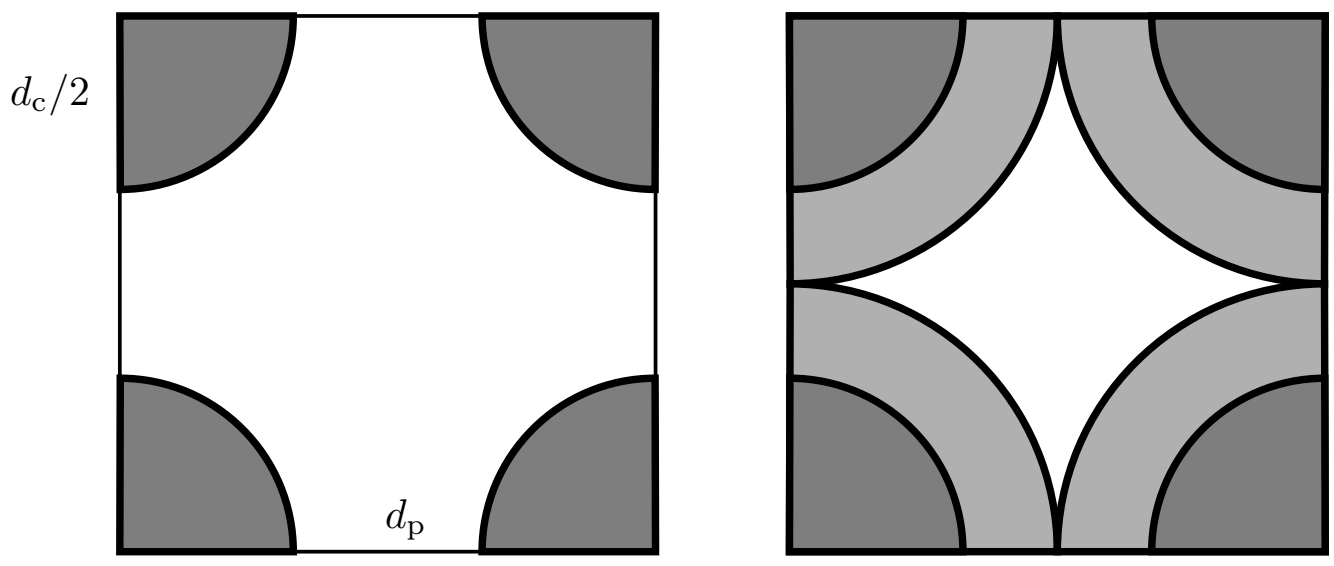

Figure 3.3. Schematic of trapping water by freezing of square lattice. Region labeling is the same as in Fig. 3.2.

Next we repeat the analysis for a square array, as shown in Fig. 3.3. The square has side length $d_{\mathrm{p}}+d_{\mathrm{c}}$; hence its area is given by

$$
A_{\square}=\left(d_{\mathrm{p}}+d_{\mathrm{c}}\right)^{2} .
$$

Taken together, the sectors form a circle of diameter $d_{\mathrm{c}}$ with area

$$
A_{\mathrm{c}, \square}=\pi\left(\frac{d_{\mathrm{c}}}{2}\right)^{2}=\frac{\pi d_{\mathrm{c}}^{2}}{4} .
$$

After freezing, the sectors form a semicircle of diameter $d_{\mathrm{c}}+d_{\mathrm{p}}$ with area

$$
A_{\mathrm{i}, \square}=\frac{\pi\left(d_{\mathrm{c}}+d_{\mathrm{p}}\right)^{2}}{4} .
$$


Hence the fraction $F$ of the water in the pore originally that becomes trapped is given by the following:

$$
F_{\square}=\frac{A_{\square}-A_{\mathrm{i}, \square}}{A_{\square}-A_{\mathrm{c}, \square}}=\frac{\left(d_{\mathrm{p}}+d_{\mathrm{c}}\right)^{2}-\pi\left(d_{\mathrm{c}}+d_{\mathrm{p}}\right)^{2} / 4}{\left(d_{\mathrm{p}}+d_{\mathrm{c}}\right)^{2}-\pi d_{\mathrm{c}}^{2} / 4}=\frac{\left(d_{\mathrm{p}}+d_{\mathrm{c}}\right)^{2}(4-\pi)}{4\left(d_{\mathrm{p}}+d_{\mathrm{c}}\right)^{2}-\pi d_{\mathrm{c}}^{2}} .
$$

With the values of the parameters from Appendix A, we have

$$
F_{\square}=0.284 .
$$

The same remarks regarding the symmetry apply as above. 


\section{Section 4: 1-D Heat Transfer Models}

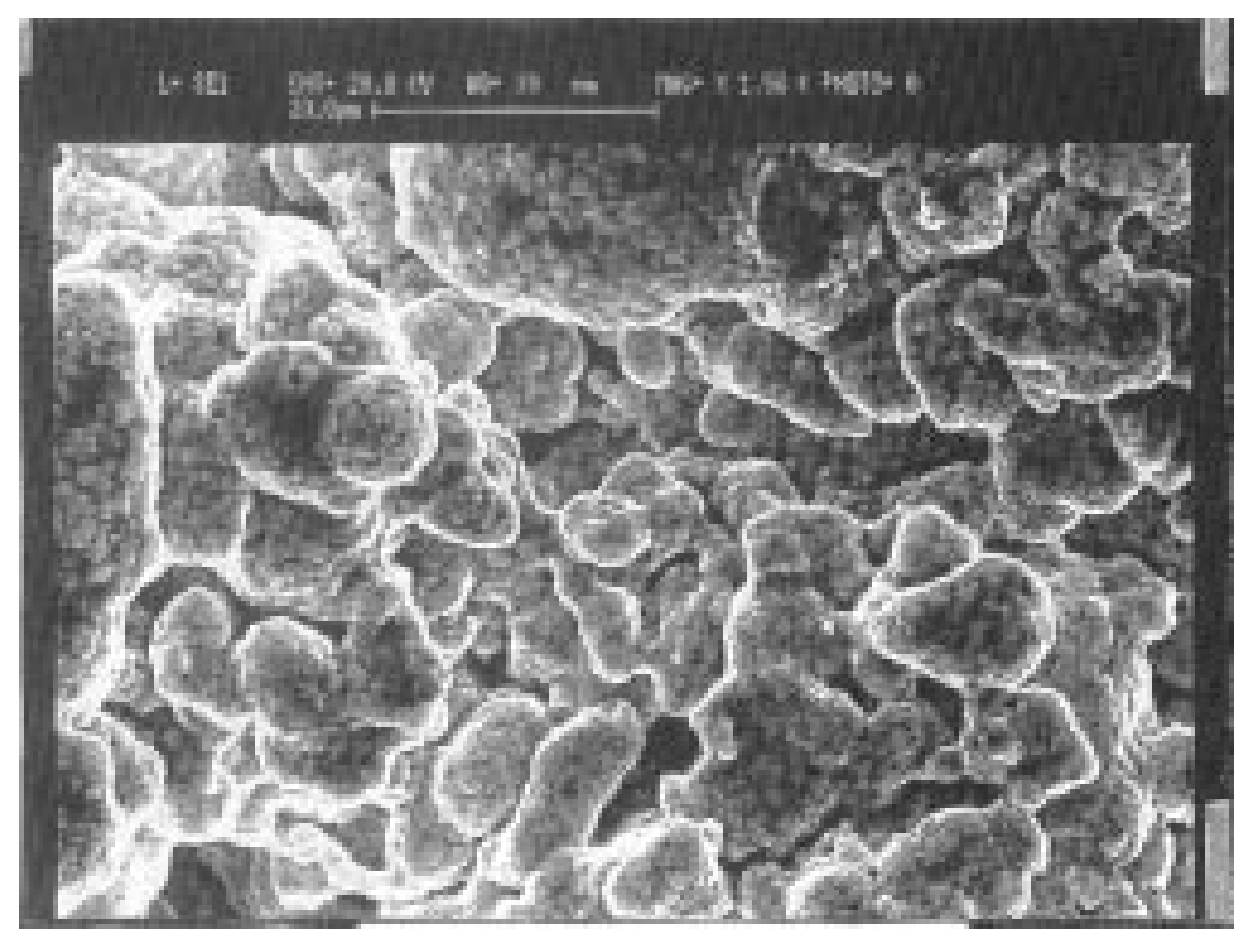

Figure 4.1. Typical microstructure of copper matrix [10].

In contrast to the description in Section 3, a more common model of porous media is to have regular geometric shapes (such as a network of cylinders) for the pores, rather than the media. This is motivated by the microstructure of the wick (see Fig. 4.1). In that case, water-filled cylinders closing up with ice would be equivalent to a pore clogging. This sort of analysis could give vastly different results about whether all the water could be squeezed out of the medium or not.

In particular, consider the scenario illustrated in Fig. 4.2. Consider two pores, $A$ and $B$. Suppose that freezing proceeds linearly along the pores. Since $A$ is more tortuous than $B$, ice from $B$ will block $A$, trapping the water in the remaining length of $A$. If this water freezes in place, it could deform the porous medium adjacent to it.

We examine some simple models to get some estimates for the time scales of the heat transfer effects. First, we consider a pore as a single cylinder which penetrates the entire wick. Its diameter would be $d_{\mathrm{p}}=50 \mu \mathrm{m}$, which is much less than the thickness of the wick, which is $7.5 \mathrm{~mm}$. Thus, the cylinder can be approximated by a one-dimensional model.

We wish to estimate the amount of time it would take for the water in the pore to freeze. At first, we consider a simple example where the water is cooled only from the outer surface of the wick. This leads to consideration of the one-phase Stefan problem where water freezes into ice. The details of the derivation may be found in [1]; we outline the steps below. 


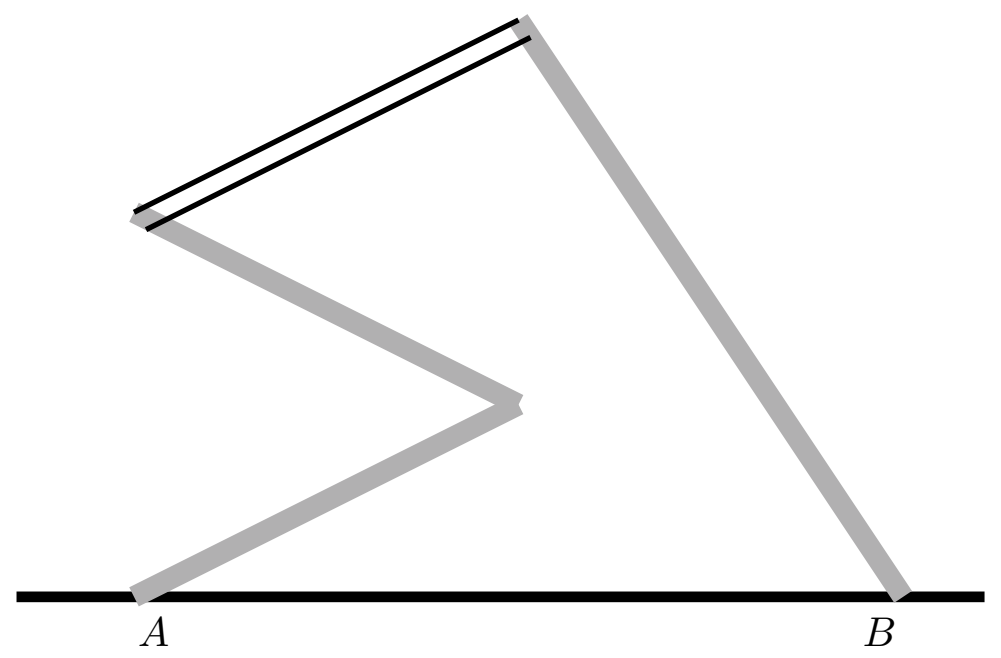

Figure 4.2. Pore structure. Grey: ice. White: water.

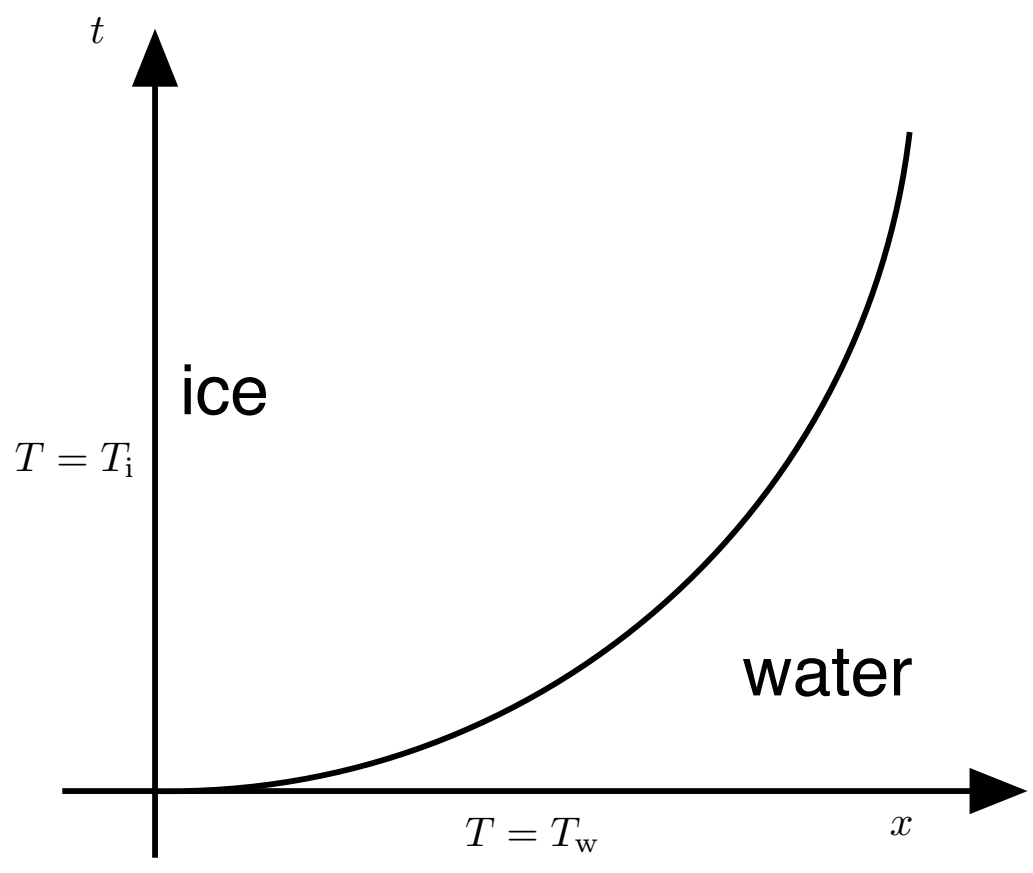

Figure 4.3. Schematic of Stefan problem.

Let $x$ be distance along the pore as measured from the outer wall of the wick (see Fig. 4.3). We consider a semi-infinite problem; this is a reasonable simplification since we are trying to get only an estimate of the freezing front progression. $T_{\mathrm{i}}(x, t)$, the temperature of the ice, follows the standard heat equation:

$$
\frac{\partial T_{\mathrm{i}}}{\partial t}=D_{\mathrm{i}} \frac{\partial^{2} T_{\mathrm{i}}}{\partial x^{2}}, \quad D_{\mathrm{i}}=\frac{k_{\mathrm{i}}}{\rho c_{\mathrm{i}}},
$$

where $c_{\mathrm{i}}$ is the specific heat and $k_{\mathrm{i}}$ is the thermal conductivity of the ice. $D_{\mathrm{i}}$ is called the thermal diffusivity (note it is usually denoted by $\alpha$, but we use $D$ since we have used $\alpha$ for 
the linear expansion factor).

Note that we have not put a subscript on the density, even though we know there is a drastic change in the density as the water freezes. The Stefan problem can be formulated with a change in density, though the setup is more complicated $[1, \S 2.3]$. Hence we proceed with a constant density (namely the one for water at $0.01^{\circ} \mathrm{C}$ ) to follow the standard Stefanproblem analysis.

The domain of (4.1) is $0<x<s(t)$, where $s(t)$ is the freezing boundary. At that boundary, we have

$$
\begin{aligned}
T_{\mathrm{i}}(s(t), t) & =0, \\
k_{\mathrm{i}} \frac{\partial T_{\mathrm{i}}}{\partial x}(s(t), t) & =\rho L \frac{d s}{d t},
\end{aligned}
$$

where $L$ is the latent heat of fusion (freezing) of water. Equation (4.2b) says that the net heat flux into the freezing front will be used up in freezing the ice into its crystalline shape. Note that in the one-phase Stefan problem, the temperature in the water is assumed to be held at exactly 0 . Hence there is no contribution from heat flux in the water to (4.2b).

We close the system with a boundary condition on $T_{\mathrm{i}}$ and an initial condition on $s$ :

$$
\begin{gathered}
T_{\mathrm{i}}(0, t)=T_{\mathrm{i} 0}, \\
s(0)=0 .
\end{gathered}
$$

With these conditions, (4.1) has a similarity solution of the form

$$
T_{\mathrm{i}}(x, t)=\Theta_{\mathrm{i}}(\zeta), \quad \zeta=\frac{x}{2 \sqrt{D_{\mathrm{i}} t}} ; \quad s(t)=2 s_{0} \sqrt{\overline{D_{\mathrm{i}} t}}
$$

Note that (4.4) automatically satisfies (4.3b).

Substituting (4.4) into (4.1) and solving, we have

$$
\begin{aligned}
-\frac{x}{4 \sqrt{D_{\mathrm{i}} t^{3}}} \frac{d \Theta_{\mathrm{i}}}{d \zeta} & =D_{\mathrm{i}} \frac{1}{4 D_{\mathrm{i}} t} \frac{d^{2} \Theta_{\mathrm{i}}}{d \zeta^{2}} \\
-2 \zeta \frac{d \Theta_{\mathrm{i}}}{d \zeta} & =\frac{d^{2} \Theta_{\mathrm{i}}}{d \zeta^{2}} \\
\frac{d \Theta_{\mathrm{i}}}{d \zeta} & =B_{1} e^{-\zeta^{2}} \\
\Theta_{\mathrm{i}}(\zeta) & =B_{1} \operatorname{erf} \zeta+B_{2} .
\end{aligned}
$$

Substituting (4.4) into (4.2a) and (4.3a) and using the resulting conditions to solve for the $B_{i}$, we obtain

$$
\begin{aligned}
\Theta_{\mathrm{i}}\left(s_{0}\right) & =0 \\
\Theta_{\mathrm{i}}(0) & =T_{\mathrm{i} 0} \\
\Theta_{\mathrm{i}}(\zeta) & =T_{\mathrm{i} 0}\left(1-\frac{\operatorname{erf} \zeta}{\operatorname{erf} s_{0}}\right)
\end{aligned}
$$


Susbtituting (4.4) into (4.2b), we have the following:

$$
\begin{aligned}
\frac{k_{\mathrm{i}}}{2 \sqrt{D_{\mathrm{i}} t}} \frac{d \Theta}{d \zeta}\left(s_{0}\right) & =\rho L s_{0} \sqrt{\frac{D_{\mathrm{i}}}{t}} \\
\frac{k_{\mathrm{i}}}{2 D_{\mathrm{i}} \rho L} \frac{d \Theta}{d \zeta}\left(s_{0}\right)=\frac{c_{\mathrm{i}}}{2 L} \frac{d \Theta}{d \zeta}\left(s_{0}\right) & =s_{0},
\end{aligned}
$$

where we have used the definition of $D_{\mathrm{i}}$ in (4.1). Substituting (4.7) into (4.8), we obtain

$$
\begin{aligned}
\frac{c_{\mathrm{i}}}{2 L}\left(-\frac{2 T_{\mathrm{i} 0}}{\sqrt{\pi}} \frac{e^{-s_{0}^{2}}}{\operatorname{erf} s_{0}}\right) & =s_{0} \\
\frac{\mathrm{St}}{\sqrt{\pi}} \frac{e^{-s_{0}^{2}}}{\operatorname{erf} s_{0}} & =s_{0}, \quad \mathrm{St}=\frac{c_{\mathrm{i}}\left|T_{\mathrm{i} 0}\right|}{L},
\end{aligned}
$$

where we have used the fact that $T_{\mathrm{i} 0}$ must be less than 0 for the ice to freeze.

In this case we take $T_{\mathrm{i} 0}=-20^{\circ} \mathrm{C}$. Then solving (4.9) numerically using the values in Appendix A, we have that

$$
s_{0}=0.237 \text {. }
$$

To traverse the wick, the freezing front must advance a distance $R_{\mathrm{m}}-R_{\mathrm{v}}$, where $R_{\mathrm{m}}$ is the outer radius of the wick (the subscript "m" refers to "middle"), while $R_{\mathrm{v}}$ is the outer radius of the core (the subscript "v" refers to "vacuum"). See Fig. 1.2. Hence the freezing front reaches the inner edge of the wick when

$$
\begin{aligned}
\left(R_{\mathrm{m}}-R_{\mathrm{v}}\right) & =2 s_{0} \sqrt{D_{\mathrm{i}} t} \\
t & =\frac{\left(R_{\mathrm{m}}-R_{\mathrm{v}}\right)^{2}}{4 s_{0}^{2} D_{\mathrm{i}}} .
\end{aligned}
$$

Then using the parameters in Appendix A, we have

$$
\begin{aligned}
t & =\frac{(2 \mathrm{~cm}-1.25 \mathrm{~cm})^{2}}{4(0.237)\left(1.23 \times 10^{-6} \mathrm{~m}^{2} / \mathrm{s}\right)}=\frac{\left(7.5 \times 10^{-3} \mathrm{~m}\right)^{2}}{4(0.237)^{2}\left(1.23 \times 10^{-6} \mathrm{~m}^{2} / \mathrm{s}\right)} \\
& =\frac{(7.5)^{2}}{4(0.237)^{2}(1.23)} \mathrm{s}=204 \mathrm{~s} .
\end{aligned}
$$

As a correction, we now examine the two-phase Stefan problem, where we have water and ice. The ice problem holds on the same domain as before, so the solution is of the form (4.7). The standard two-phase Stefan condition states that the difference in the fluxes propels the front:

$$
k_{\mathrm{i}} \frac{\partial T_{\mathrm{i}}}{\partial x}\left(s^{-}(t), t\right)-k_{\mathrm{w}} \frac{\partial T_{\mathrm{w}}}{\partial x}\left(s^{+}(t), t\right)=\rho L \frac{d s}{d t} .
$$

Equation (4.12) replaces $(4.2 \mathrm{~b})$ in our analysis. Note also that in the limit where the water temperature is identically zero, the flux in the water region vanishes and we obtain (4.2b). Also, in the water we have an initial condition, rather than a boundary condition:

$$
T_{\mathrm{w}}(x, 0)=T_{\mathrm{w} 0}
$$


We may again use the similarity form (4.4), but we have to be careful to keep track of the different quantities in the ice and water. In particular, for the water temperature, (4.5a) is replaced by

$$
\begin{aligned}
-\frac{x}{4 \sqrt{D_{\mathrm{i}} t^{3}}} \frac{d \Theta_{\mathrm{w}}}{d \zeta} & =D_{\mathrm{w}} \frac{1}{4 D_{\mathrm{i}} t} \frac{d^{2} \Theta_{\mathrm{w}}}{d \zeta^{2}} \\
-2 \zeta \frac{D_{\mathrm{i}}}{D_{\mathrm{w}}} \frac{d \Theta_{\mathrm{w}}}{d \zeta} & =\frac{d^{2} \Theta}{d \zeta^{2}} \\
\frac{d \Theta_{\mathrm{w}}}{d \zeta} & =B_{1} \exp \left(-\frac{D_{\mathrm{i}} \zeta^{2}}{D_{\mathrm{w}}}\right) \\
\Theta_{\mathrm{w}}(\zeta) & =B_{1} \operatorname{erfc}\left(\zeta \sqrt{\frac{D_{\mathrm{i}}}{D_{\mathrm{w}}}}\right)+B_{2},
\end{aligned}
$$

where we use the complementary error function for algebraic convenience. Our boundary condition (4.13) becomes

$$
\Theta_{\mathrm{w}}(\infty)=T_{\mathrm{w} 0} .
$$

Equation (4.6a) holds for $\Theta_{\mathrm{w}}$ as well, since at $s(t)$ the temperature must be continuous at the freezing temperature. Solving (4.14) subject to this fact and (4.15), we obtain

$$
\Theta_{\mathrm{w}}(\zeta)=T_{\mathrm{w} 0}\left[1-\frac{\operatorname{erfc}\left(\zeta \sqrt{D_{\mathrm{i}} / D_{\mathrm{w}}}\right)}{\operatorname{erfc}\left(s_{0} \sqrt{D_{\mathrm{i}} / D_{\mathrm{w}}}\right)}\right] .
$$

Substituting (4.4) into (4.12), we have the following:

$$
\begin{aligned}
& \frac{k_{\mathrm{i}}}{2 \sqrt{D_{\mathrm{i} t}}} \frac{d \Theta_{\mathrm{i}}}{d \zeta}\left(s_{0}\right)-\frac{k_{\mathrm{w}}}{2 \sqrt{D_{\mathrm{i}} t}} \frac{d \Theta_{\mathrm{w}}}{d \zeta}\left(s_{0}\right)=\rho L s_{0} \sqrt{\frac{D_{\mathrm{i}}}{t}} \\
& \frac{k_{\mathrm{i}}}{2 D_{\mathrm{i}} \rho L} \frac{d \Theta_{\mathrm{i}}}{d \zeta}\left(s_{0}\right)-\frac{k_{\mathrm{w}}}{2 D_{\mathrm{i}} \rho L} \frac{d \Theta_{\mathrm{w}}}{d \zeta}\left(s_{0}\right)=s_{0} .
\end{aligned}
$$

Substituting (4.7) and (4.16) into (4.17), we obtain:

$$
\begin{array}{r}
\frac{c_{\mathrm{i}}}{2 L}\left(-\frac{2 T_{\mathrm{i} 0}}{\sqrt{\pi}} \frac{e^{-s_{0}^{2}}}{\operatorname{erf} s_{0}}\right)-\frac{k_{\mathrm{w}}}{2 D_{\mathrm{i}} \rho L}\left[\frac{2 T_{\mathrm{w} 0}}{\sqrt{\pi}} \sqrt{\frac{D_{\mathrm{i}}}{D_{\mathrm{w}}}} \frac{e^{-D_{\mathrm{i}} s_{0}^{2} / D_{\mathrm{w}}}}{\operatorname{erfc}\left(s_{0} \sqrt{D_{\mathrm{i}} / D_{\mathrm{w}}}\right)}\right]=s_{0} \\
\frac{c_{\mathrm{i}}}{L}\left(-\frac{T_{\mathrm{i} 0}}{\sqrt{\pi}} \frac{e^{-s_{0}^{2}}}{\operatorname{erf} s_{0}}\right)-\frac{k_{\mathrm{w}}}{2 \rho L D_{\mathrm{w}}} \sqrt{\frac{D_{\mathrm{w}}}{D_{\mathrm{i}}}}\left[\frac{2 T_{\mathrm{w} 0}}{\sqrt{\pi}} \frac{e^{-D_{\mathrm{i}} s_{0}^{2} / D_{\mathrm{w}}}}{\operatorname{erfc}\left(s_{0} \sqrt{D_{\mathrm{i}} / D_{\mathrm{w}}}\right)}\right]=s_{0} \\
\frac{\mathrm{St}_{\mathrm{i}}}{\sqrt{\pi}} \frac{e^{-s_{0}^{2}}}{\operatorname{erf} s_{0}}-\frac{c_{\mathrm{w}} T_{\mathrm{w} 0}}{L \sqrt{\pi}} \sqrt{\frac{D_{\mathrm{w}}}{D_{\mathrm{i}}}} \frac{e^{-D_{\mathrm{i}} s_{0}^{2} / D_{\mathrm{w}}}}{\operatorname{erfc}\left(s_{0} \sqrt{D_{\mathrm{i}} / D_{\mathrm{w}}}\right)}=s_{0} \\
\frac{\mathrm{St}_{\mathrm{i}}}{\sqrt{\pi}} \frac{e^{-s_{0}^{2}}}{\operatorname{erf} s_{0}}-\frac{\mathrm{St}_{\mathrm{w}}}{\sqrt{\pi}} \sqrt{\frac{D_{\mathrm{w}}}{D_{\mathrm{i}}}} \frac{e^{-D_{\mathrm{i}} s_{0}^{2} / D_{\mathrm{w}}}}{\operatorname{erfc}\left(s_{0} \sqrt{D_{\mathrm{i}} / D_{\mathrm{w}}}\right)}=s_{0}, \quad \mathrm{St}_{\mathrm{w}}=\frac{c_{\mathrm{w}} T_{\mathrm{w} 0}}{L} .
\end{array}
$$


In this case we take $T_{\mathrm{w} 0}=20^{\circ} \mathrm{C}$. Then solving (4.18) using the values in Appendix A, we have that

$$
s_{0}=0.200 \text {. }
$$

Note that the front speed is lower, which is expected since now the copper has to cool the water from room temperature before freezing it. Substituting (4.19) into (4.11a), we have

$$
t=\frac{(7.5)^{2}}{4(0.200)^{2}(1.23)} \mathrm{s}=287 \mathrm{~s}
$$

which is longer, also as expected.

However, both of these times seem long, especially given that the cross-sectional area of the pore is so small. But recall that when using the one-dimensional approximation, we are essentially assuming that the only heat sink is at the wick exit. In other words, we are treating the sides of the pore as insulating, when they are really conducting.

Therefore, the next estimate we compute is how long it takes the copper wick material to cool once the pipe is exposed to a cold temperature. This is a simpler problem, since there is no phase change. Hence the heat equation (4.1) still holds with $D_{\mathrm{i}}$ replaced by $D_{\text {c }}$, the thermal diffusivity of copper.

To make the problem simple, we assume an infinite domain, so a similarity solution still holds. Therefore, we solve (4.1) subject to (4.3a) and (4.13), which corresponds to substituting the functional form in (4.5b) into (4.6b) and (4.15). Our solution is given by

$$
\Theta_{\mathrm{c}}(\zeta)=T_{\mathrm{w} 0}+\left(T_{\mathrm{i} 0}-T_{\mathrm{w} 0}\right) \operatorname{erfc} \zeta, \quad \zeta=\frac{x}{2 \sqrt{D_{\mathrm{c}} t}} .
$$

In this case $x=0$ corresponds to the outside of the pipe (radius $R_{\mathrm{h}}$, where the subscript "h" refers to "heat pipe"), since that is where the copper is exposed to the external cold temperature (see Fig. 1.2). To get an estimate of the time for heat transport, we find the time when the temperature at the inside of the wick has evolved to within $5 \%$ of equilibrium. In other words:

$$
\begin{aligned}
T_{\mathrm{c}}\left(R_{\mathrm{h}}-R_{\mathrm{v}}, t\right) & =T_{\mathrm{w} 0}+\left(T_{\mathrm{i} 0}-T_{\mathrm{w} 0}\right)(0.95) \\
T_{\mathrm{w} 0}+\left(T_{\mathrm{i} 0}-T_{\mathrm{w} 0}\right) \operatorname{erfc}\left(\frac{R_{\mathrm{h}}-R_{\mathrm{v}}}{2 \sqrt{D_{\mathrm{c}} t}}\right) & =T_{\mathrm{w} 0}+\left(T_{\mathrm{i} 0}-T_{\mathrm{w} 0}\right)(0.95) \\
\operatorname{erfc}\left(\frac{R_{\mathrm{h}}-R_{\mathrm{v}}}{2 \sqrt{D_{\mathrm{c}} t}}\right) & =0.95 \\
\frac{R_{\mathrm{h}}-R_{\mathrm{v}}}{2 \sqrt{D_{\mathrm{c}} t}} & =4.43 \times 10^{-2},
\end{aligned}
$$

where we have used our new definition of $\zeta$. Using the parameters in Appendix A, we have that

$$
t=\frac{(12.5)^{2}}{4\left(4.43 \times 10^{-2}\right)^{2}(116)} \mathrm{s}=171 \mathrm{~s} .
$$

Note this is less than the time given in (4.11b) and (4.20). It's not that much less, even though the thermal diffusivity of copper is much higher than water. The reason is because in the case of water, we were tracking cooling down to $0^{\circ} \mathrm{C}$, while in the case of copper we are treating cooling down to $-18^{\circ} \mathrm{C}$ (95\% of the total change in temperature). 


\section{Section 5: Other Heat Transfer Models}

If the copper gets colder faster than the water, one may think of tracking freezing in the cross-section of the pore, rather than longitudinally. In particular, we consider the heat equation in a circular cross-section. We assume that the temperature at the outer edge is uniform at $T_{\mathrm{i} 0}$. Hence there is no angular dependence on the problem and the heat equation becomes

$$
\frac{\partial T_{\mathrm{w}}}{\partial t}=\frac{D_{\mathrm{w}}}{r} \frac{\partial}{\partial r}\left(r \frac{\partial T_{\mathrm{w}}}{\partial r}\right), \quad T_{\mathrm{w}}\left(d_{\mathrm{p}} / 2, t\right)=T_{\mathrm{i} 0}, \quad T_{\mathrm{w}}(r, 0)=T_{\mathrm{w} 0} .
$$

For now, we derive a rough estimate of the time it would take the water in this situation to freeze. The time scale for the problem is given by

$$
\frac{\left(d_{\mathrm{p}} / 2\right)^{2}}{D_{\mathrm{w}}}=\frac{\left(50 \times 10^{-6} \mathrm{~m}\right)^{2}}{1.5 \times 10^{-7} \mathrm{~m}^{2} / \mathrm{s}}=\frac{2500 \times 10^{-5} \mathrm{~s}}{1.5}=1.8 \times 10^{-2} \mathrm{~s},
$$

where we have used the parameters in Appendix A.

But this is an incredibly small time. How can this be? Recall that when setting up the problem as in (5.1), we implicitly assumed that there was an infinite reservoir of cold copper at temperature $T_{\mathrm{i} 0}$. But from the parameters in Appendix A, we see that this is not the case. In particular, the size of the pores and the copper between the cores is comparable. Hence a more realistic situation is diagrammed in Fig. 5.1.

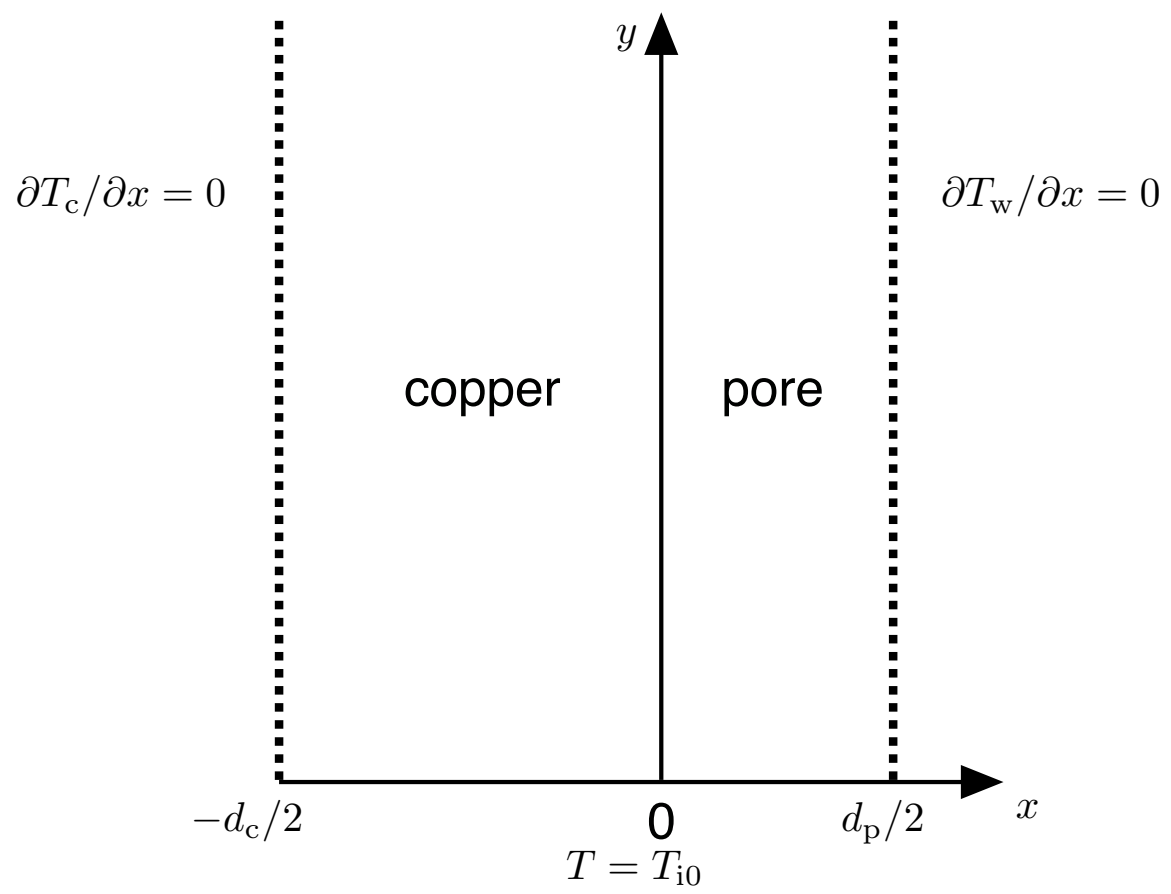

Figure 5.1. Coupled pore-copper system. Dotted lines are lines of symmetry. 
We reduce to a Cartesian coordinate system. Here $x$ is along the cross-section of the pore, while $y$ measures along the pore (which we take to be semi-infinite). We take $x=0$ to be the pore-copper interface. We also take a periodic array of copper and pore, so there are lines of symmetry at $x=-d_{\mathrm{c}} / 2$ and $x=d_{\mathrm{p}} / 2$.

The governing equations are as below. First, the heat equation (with the appropriate thermal diffusivity) holds in each region:

$$
\begin{array}{cl}
\frac{\partial T_{\mathrm{c}}}{\partial t}=D_{\mathrm{c}}\left(\frac{\partial^{2} T_{\mathrm{c}}}{\partial x^{2}}+\frac{\partial^{2} T_{\mathrm{c}}}{\partial y^{2}}\right), & -d_{\mathrm{c}} / 2 \leq x \leq 0, \quad y>0, \\
\frac{\partial T_{\mathrm{w}}}{\partial t}=D_{\mathrm{w}}\left(\frac{\partial^{2} T_{\mathrm{w}}}{\partial x^{2}}+\frac{\partial^{2} T_{\mathrm{w}}}{\partial y^{2}}\right), \quad 0 \leq x \leq d_{\mathrm{p}} / 2, \quad y>0 .
\end{array}
$$

The bottom of each region is held at the cold-copper temperature:

$$
T_{\mathrm{c}}(x, 0, t)=T_{\mathrm{w}}(x, 0, t)=T_{\mathrm{i} 0} .
$$

Initially, the temperature is taken to be the warm-water temperature:

$$
T_{\mathrm{c}}(x, y, 0)=T_{\mathrm{w}}(x, y, 0)=T_{\mathrm{i} 0} .
$$

Since we have a periodic array, there is no flux through the outer boundaries:

$$
\frac{\partial T_{\mathrm{c}}}{\partial x}\left(x,-d_{\mathrm{c}} / 2, t\right)=\frac{\partial T_{\mathrm{w}}}{\partial x}\left(x, d_{\mathrm{p}} / 2, t\right)=0
$$

At the boundary between the two regions, the heat flux is constant:

$$
k_{\mathrm{c}} \frac{\partial T_{\mathrm{c}}}{\partial x}(x, 0, t)=k_{\mathrm{w}} \frac{\partial T_{\mathrm{w}}}{\partial x}(x, 0, t) .
$$

Equations (5.2)-(5.4) form a simple closed heat-transfer system. Since it does not include the latent-heat considerations of a full Stefan problem, it will underestimate the true time needed for the pore/copper system to freeze. Equations (5.2)-(5.4) may be solved in Matlab, but a full implementation is beyond the scope of this manuscript. 


\section{Section 6: Conclusions and Further Research}

In order for heat pipes to be used in space, designers must be confident that they can survive the freeze-thaw cycles that occur there. The pipe itself must be resistant to bursting, and the porous media structure of the sintered copper wick must resist deformation.

In this work we modeled both failure mechanisms. For pipe failure, it is hypothesized that if liquid water leaches out of the wick into the void space, it will form a pool. It is the freezing of that pool which then causes expansion and pipe failure.

We used a simple volume-balance approach to study this situation. We tracked the expansion of water and the contraction of copper during freezing. Our calculations showed that if the charge percentage of water was under $97 \%$ of the wick capacity, the frozen water would remain inside the wick, rather than leaking out into the void space. Though heat pipes for terrestrial applications are often charged higher than this, typical prototypes for space applications are charged at only $90 \%$. Hence this type of failure should be easily avoided.

The problem of failure by deformation of the wick structure was more complicated. First, we modeled the structure as a series of copper balls (or cylinders, in two dimensions) around which ice formed. We searched for pockets of water that became trapped by ice on all sides. We hypothesized that the freezing of such pockets would cause pressure that could deform the porous matrix structure.

In the case of a regular hexagonal array of copper beads, we found that the fraction of liquid water originally in the matrix that could be trapped was around $13 \%$. However, this regular structure had the highest fraction; for randomly chosen triangles, the volume would be much less (see Appendix B). A square lattice was even worse, with $28 \%$ of the volume trapped. We determined that the use of software such as Mathematica could help do these calculations when the array consisted of triangles of random side length, and perhaps could help in the harder three-dimensional case as well.

By treating the wick as a series of copper spheres whose effective radius expands with accumulating ice, we may be able to use techniques from the field of persistent homology. The advantage to looking at the problem this way is that software exists which (hopefully) could take a set of spheres with a random distribution of pore sizes between them (as in [10]), and then accumulate ice to them to track trapped water inclusions in a random matrix.

We next examined wick failure by tracking the water in individual pores. The overarching goal was to see if ice advancing in less-torturous pores could trap water in moretorturous ones. First, to get an idea of front speed, we examined one-dimensional models, where the heat sink was considered to be at one end. We solved the problem as both a oneand two-phase Stefan problem, obtaining estimates of around 3-5 minutes for the time to take ice to traverse the wick. In contrast, the time for the copper to reach the coldest temperature at the inside of the wick was estimated to be slightly less than 3 minutes.

These one-dimensional models are unrealistic, as the sintered copper is spread throughout the pore structure, and thus heat would be drawn from the water all along the pore. 
We then considered more detailed models for the pore. Considering the sides of the pore to be made of copper maintained below freezing proved to be unrealistic, leading to freezing times of milliseconds. This is because the widths of the pores and the connecting copper structures are of the same magnitude. Hence heat is conducted away from the water at a rate limited by the conduction properties of the copper.

Though we were able to write down the governing equations for such a model, a full solution is beyond the scope of this manuscript.

In summary, our calculations indicate that with current (90\%) loading for heat pipe prototypes for space use, it is unlikely that the heat pipe will rupture. This is because such loading leaves enough space in the wick after freezing so water does not accumulate in the void space (which is the theorized mechanism for pipe rupture). They also indicate that it is unlikely for the wick itself to fail. Given the connectedness of the pores in the wick, the advancing ice front should push the remaining water toward the center of the pipe without pushing it into the central void space. Moreover, it should do so without trapping appreciable amounts of water in ice pockets. 
Draft Date: August 21, 2018

\section{Acknowledgments}

We wish to thank the following people for their contributions to the project:

- Peter Kramer of Rensselaer Polytechnic Institute for helpful discussions regarding the models in Section 3.

- Ferran Planella of Oxford University, for references regarding phase changes in porous media.

- Tracy Stepien of the University of Arizona and Manuchehr Aminian of Colorado State University, for references and ideas regarding persistent homology. 


\section{Nomenclature}

Units are listed in terms of mass $(M)$, length $(L)$, temperature $(\Theta)$, and time $(T)$. If a symbol appears both with and without tildes, the symbol with tildes has units, while the one without is dimensionless. Equation numbers where a variable is first defined is listed, if appropriate.

A: area, units $L^{2}(3.1)$.

$B$ : arbitrary constant, variously defined.

$c$ : specific heat, units $L^{2} / T^{2} \Theta(4.1)$.

$D$ : thermal diffusivity, units $L^{2} / T(4.1)$.

$d$ : diameter of pore or particle, units $L(3.1)$.

$F$ : trapped water ratio (3.4).

$f$ : charging factor.

$k$ : thermal conductivity, units $M L / T^{3} \Theta(4.1)$.

$L$ : latent heat of fusion, units $L^{2} / T^{2}(4.2 \mathrm{~b})$.

$R$ : radial measurement, units $L$ (4.11a).

$r$ : radius of pore or particle, units $L$ (5.1).

St: Stefan number (4.9).

$s(t)$ : freezing boundary, units $L(4.2 \mathrm{a})$.

$T$ : temperature, units $\Theta$.

$t$ : time, units $T$ (4.1).

$x$ : distance along pore, units $L$ (4.1).

$y$ : distance across pore or copper, units $L$ (5.2a).

$V_{\mathrm{r}}$ : volumetric expansion factor (2.1).

$\alpha$ : linear expansion factor.

$\zeta$ : similarity variable (4.4).

$\Theta(\zeta)$ : similarity solution for temperature, units $\Theta(4.4)$.

$\rho$ : density, units $M / L^{3}(2.1)$.

$\phi$ : void fraction.

\section{Other Notation}

c: as a subscript, used to indicate copper (3.1).

h: as a subscript on $R$, used to indicate the heat pipe (4.22).

i: as a subscript, used to indicate ice.

$\mathrm{m}$ : as a subscript on $R$, used to indicate the wick (4.11a).

p: as a subscript on $d$, used to indicate a pore (3.1).

$\mathrm{v}$ : as a subscript on $R$, used to indicate the inner wick radius (4.11a). 
w: as a subscript, used to indicate water.

0: as a subscript, used to indicate an initial value. 
Draft Date: August 21, 2018

\section{References}

[1] V. Alexiades and A. D. Solomon. Mathematical Modeling Of Melting And Freezing Processes. Taylor \& Francis, 1992.

[2] Capri. Sintered wick structured heat pipes. URL: http://capri.co.in/ sintered-wick-structured-heat-pipes/ [cited June 26, 2018].

[3] Kwok Cheung. Flight qualification of copper water heat pipes at naval research laboratory. In 38th AIAA Thermophysics Conference. American Institute of Aeronautics and Astronautics, 2005.

[4] F. Cverna and ASM International. Materials Properties Database Committee. ASM ready reference: Thermal properties of metals. Materials data series. ASM International, 2002.

[5] Engineering Toolbox. Ice - thermal properties. URL: https://www . engineeringtoolbox.com/ice-thermal-properties-d_576.html [cited June 26, 2018].

[6] Engineering Toolbox. Water - density, specific weight and thermal expansion coefficient. URL: https://www.engineeringtoolbox.com/ water-density-specific-weight-d_595.html [cited June 26, 2018].

[7] Engineering

Toolbox. Water - specific heat. URL: https://www.engineeringtoolbox. com/specific-heat-capacity-water-d_660.html?vA=20\&units=C\# [cited June 28, 2018].

[8] Engineering Toolbox. Water - thermal conductivity. URL: https://www.engineeringtoolbox.com/ water-liquid-gas-thermal-conductivity-temperature-pressure-d_2012.html [cited June 28, 2018].

[9] Panayiotis J Karditsas and Marc-Jean Baptiste. Pure copper. URL: http:// wWw-ferp.ucsd.edu/LIB/PROPS/PANOS/cu.html [cited July 6, 2018].

[10] K.C. Leong, C.Y. Liu, and Max Lu. Characterization of sintered copper wicks used in heat pipes. Journal of Porous Materials, 4:303-308, 1997.

[11] Wikipedia. Latent heat. URL: https://en.wikipedia.org/wiki/Latent_heat [cited June 28, 2018]. 
Draft Date: August 21, 2018

\section{Appendix A: Parameter Values}

The linear expansion factor for cast copper is given by $[4$, p. 11]:

$$
\frac{17 \times 10^{-6}}{\mathrm{~K}} \leq \alpha \leq \frac{18 \times 10^{-6}}{\mathrm{~K}}
$$

and so we take the midpoint of the range:

$$
\alpha=\frac{1.75 \times 10^{-5}}{\circ \mathrm{C}},
$$

where we have used the fact that a differential degree Celsius is the same as a differential Kelvin.

The density of water at room temperature is given by [6]:

$$
\rho_{\mathrm{w}}=998.21 \frac{\mathrm{kg}}{\mathrm{m}^{3}}
$$

while the density of ice at $0^{\circ} \mathrm{C}$ is given by [5]:

$$
\rho_{\mathrm{i}}=916.2 \frac{\mathrm{kg}}{\mathrm{m}^{3}} .
$$

Substituting these values into (2.1), we have the following for the volumetric expansion coefficient:

$$
V_{\mathrm{r}}=\frac{998.21}{916.2}=1.090 \text {. }
$$

According to [10], the typical diameter of the copper powder (bead) is

$$
d_{\mathrm{c}}=63 \mu \mathrm{m},
$$

and the typical pore diameter is

$$
d_{\mathrm{p}}=50 \mu \mathrm{m} \text {. }
$$

At $-20^{\circ} \mathrm{C}$, the properties of ice are given by [5]:

$$
k_{\mathrm{i}}=2.39 \frac{\mathrm{W}}{\mathrm{m} \cdot \mathrm{K}}, \quad c_{\mathrm{i}}=1.943 \frac{\mathrm{kJ}}{\mathrm{kg} \cdot \mathrm{K}},
$$

while the density of water at $0.01^{\circ} \mathrm{C}$ is given by [5]:

$$
\rho=999.8 \frac{\mathrm{kg}}{\mathrm{m}^{3}} .
$$


Using these values in (4.1), we have the following:

$$
\begin{aligned}
D_{\mathrm{i}} & =\left(2.39 \frac{\mathrm{W}}{\mathrm{m} \cdot \mathrm{K}}\right)\left(999.8 \frac{\mathrm{kg}}{\mathrm{m}^{3}}\right)^{-1}\left(1.943 \frac{\mathrm{kJ}}{\mathrm{kg} \cdot \mathrm{K}}\right)^{-1}=\frac{2.39 \mathrm{~J} / \mathrm{s} \cdot \mathrm{m}^{2}}{(999.8)\left(1.943 \times 10^{3} \mathrm{~J}\right)} \\
& =1.23 \times 10^{-6} \frac{\mathrm{m}^{2}}{\mathrm{~s}} .
\end{aligned}
$$

Moreover, we have that the latent heat of fusion for ice is given by [11]:

$$
L=334 \frac{\mathrm{kJ}}{\mathrm{kg}} .
$$

Substituting these values into (4.9), we have that (using $T_{\mathrm{i} 0}=-20$ )

$$
\mathrm{St}_{\mathrm{i}}=(20 \mathrm{~K})\left(1.943 \frac{\mathrm{kJ}}{\mathrm{kg} \cdot \mathrm{K}}\right)\left(334 \frac{\mathrm{kJ}}{\mathrm{kg}}\right)^{-1}=\frac{(20)(1.943)}{334}=0.116 .
$$

At $20^{\circ} \mathrm{C}$, the properties of water are given by [7], [8]:

$$
k_{\mathrm{w}}=598.03 \frac{\mathrm{mW}}{\mathrm{m} \cdot \mathrm{K}}, \quad c_{\mathrm{w}}=4.1844 \frac{\mathrm{kJ}}{\mathrm{kg} \cdot \mathrm{K}} .
$$

Substituting (A.5b) and (A.9) into (4.1), we have that

$$
\begin{aligned}
D_{\mathrm{w}} & =\left(598.03 \frac{\mathrm{mW}}{\mathrm{m} \cdot \mathrm{K}}\right)\left(999.8 \frac{\mathrm{kg}}{\mathrm{m}^{3}}\right)^{-1}\left(4.1844 \frac{\mathrm{kJ}}{\mathrm{kg} \cdot \mathrm{K}}\right)^{-1}=\frac{0.59803 \mathrm{~J} / \mathrm{s} \cdot \mathrm{m}^{2}}{(999.8)\left(4.1844 \times 10^{3} \mathrm{~J}\right)} \\
& =1.5 \times 10^{-7} \frac{\mathrm{m}^{2}}{\mathrm{~s}} .
\end{aligned}
$$

Given these values, we may use (4.18) to calculate $\mathrm{St}_{\mathrm{w}}$ (using $T_{\mathrm{w} 0}=20$ ):

$$
\mathrm{St}_{\mathrm{w}}=(20 \mathrm{~K})\left(4.1844 \frac{\mathrm{kJ}}{\mathrm{kg} \cdot \mathrm{K}}\right)\left(334 \frac{\mathrm{kJ}}{\mathrm{kg}}\right)^{-1}=\frac{(20)(4.1844)}{334}=0.25 .
$$

The outer radius of the heat pipe is given by

$$
R_{\mathrm{h}}=2.5 \mathrm{~cm}
$$

while the outer radius of the wick is given by

$$
R_{\mathrm{m}}=2 \mathrm{~cm} \text {. }
$$

(Note that this is also the inner radius of the pipe wall.) The inner radius of the wick is given by

$$
R_{\mathrm{v}}=1.25 \mathrm{~cm} \text {. }
$$

The properties of copper are given by [9]:

$$
k_{\mathrm{c}}=401 \frac{\mathrm{W}}{\mathrm{m} \cdot \mathrm{K}}, \quad c_{\mathrm{w}}=385 \frac{\mathrm{J}}{\mathrm{kg} \cdot \mathrm{K}}, \quad \rho_{\mathrm{c}}=8.96 \frac{\mathrm{g}}{\mathrm{cm}^{3}} .
$$

Substituting (A.13) into (4.1), we have the following:

$$
\begin{aligned}
D_{\mathrm{c}} & =\left(401 \frac{\mathrm{W}}{\mathrm{m} \cdot \mathrm{K}}\right)\left(8.96 \frac{\mathrm{g}}{\mathrm{cm}^{3}}\right)^{-1}\left(385 \frac{\mathrm{J}}{\mathrm{kg} \cdot \mathrm{K}}\right)^{-1}=\frac{401}{(8.96)(385)} \frac{\mathrm{J} \cdot \mathrm{cm}^{3} \cdot \mathrm{kg}}{\mathrm{m} \cdot \mathrm{s} \cdot \mathrm{g} \cdot \mathrm{J}} \\
& =0.116 \frac{\left(10^{-6} \mathrm{~m}^{3}\right)\left(10^{3} \mathrm{~g}\right)}{\mathrm{m} \cdot \mathrm{s} \cdot \mathrm{g}}=1.16 \times 10^{-4} \frac{\mathrm{m}^{2}}{\mathrm{~s}} .
\end{aligned}
$$




\section{Appendix B: Using Mathematica to Calculate Percentage Trapped \\ Volume}

\section{Before Freezing}

First we start with a series of points.

$\operatorname{In}[\cdot]:=$ Vertices $=\{\{0,0\},\{1,3\},\{3,0\}\}$

Out $[\cdot]=\left(\begin{array}{ll}0 & 0 \\ 1 & 3 \\ 3 & 0\end{array}\right)$

From these vertices we construct the three sides, and find the maximum length. For illustration, we use $1 / 4$ of this length as the radius of the copper disks:

$\ln [\cdot]:=$ Sides $=\{$ Line $[\{$ Vertices $[[1]]$, Vertices $[[2]]\}]$,

Line $[\{\operatorname{Vertices}[[1]], \operatorname{Vertices}[[3]]\}]$, Line $[\{\operatorname{Vertices}[[3]], \operatorname{Vertices}[[2]]\}]\}$;

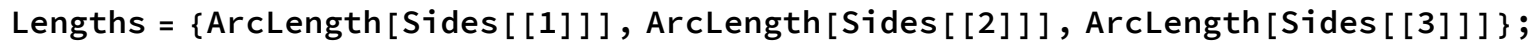

minrad $=\operatorname{Max}[$ Lengths $] / 4$

Out $[\cdot]=\frac{\sqrt{13}}{4}$

Now we can illustrate the situation. 

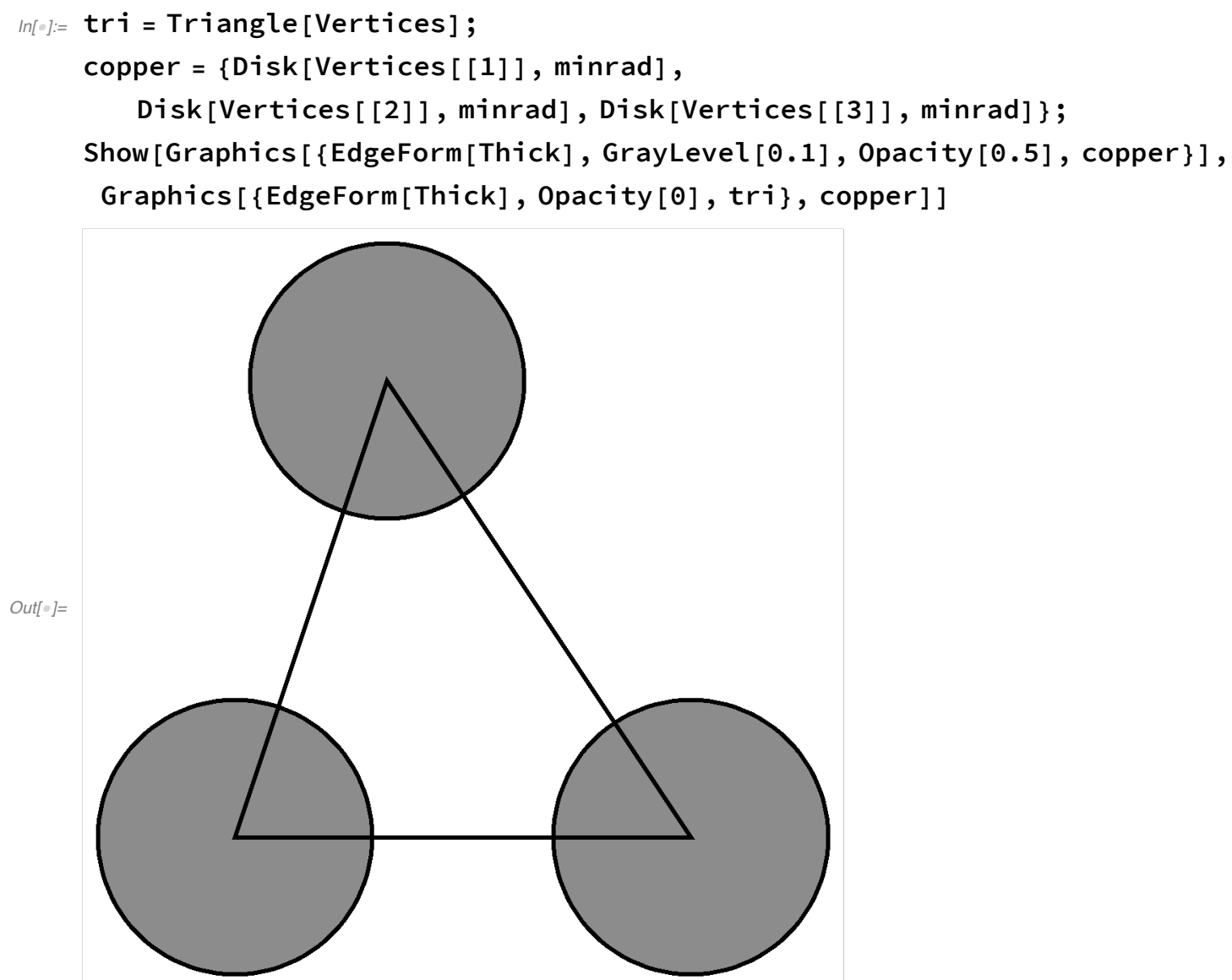

The area of the water originally is the area of the triangle less the grey region. Since the central angles sum to $\pi$, the area of the sectors is half the area of one of the circles.

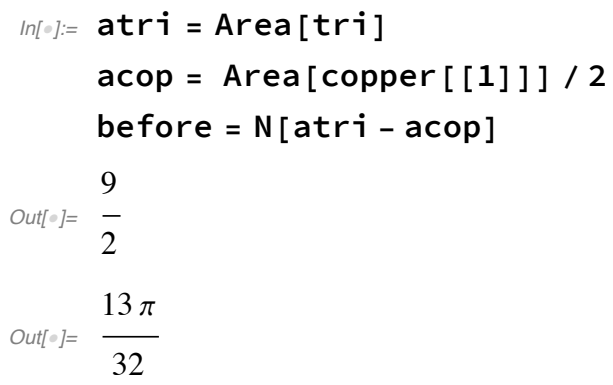

\section{After Freezing}

In this case the maximum length is the diameter of the desired disks:

$\ln [\cdot]:=\max r a d=\operatorname{Max}[$ Lengths $] / 2$

Out $[\cdot]=\frac{\sqrt{13}}{2}$

Now we can illustrate the situation. 
$\ln [\cdot]:=$ ice $=\{$ Disk $[$ Vertices $[[1]], \operatorname{maxrad}]$,

Disk[Vertices [ [2] ], maxrad], Disk[Vertices [3] ], maxrad] \};

Show [Graphics [ $\{$ EdgeForm [Thick], GrayLevel[0.1], Opacity [0.5], copper\}],

Graphics [\{EdgeForm [Thick], Gray, Opacity [0.5], ice\}],

Graphics [\{EdgeForm [Thick], Opacity[0], tri\}, ice] ]

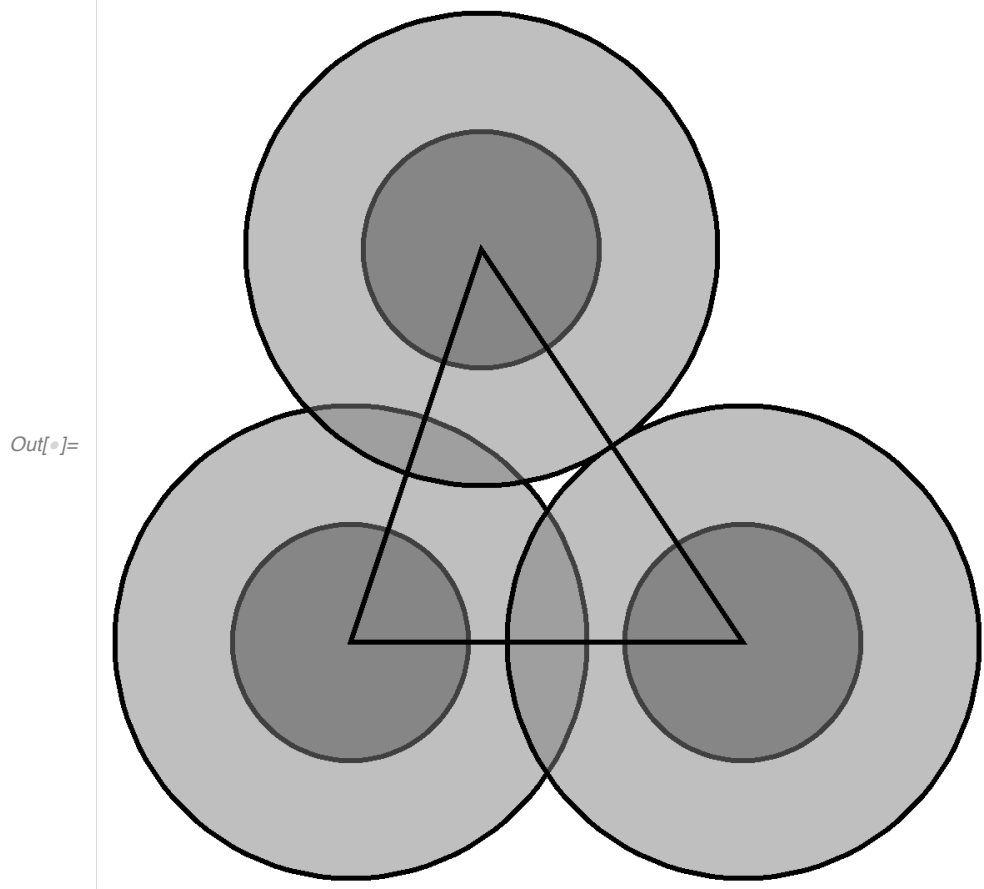

The area of the white region is given by the area of the triangle less the grey region. Since the central angles sum to $\pi$, the area of the sectors (with overlap) is half the area of one of the larger circles. The area of the overlap is half the area of the overlap of the circles in total. Hence we have

$\operatorname{In}[-]:=\operatorname{asec}=\operatorname{Area}[$ ice $[[1]]] / 2$;

over $=$

(Area [RegionIntersection [ice [ [1] ], ice [ [2] ]] ] + Area [RegionIntersection [ice [ [3] ], ice [[2] ] ] + Area [RegionIntersection [ice [ [1]], ice [ [3]]]]) / 2;

after $=\mathrm{N}[$ atri $-($ asec - over $)]$

Out $[\cdot]=0.0651663$

And then the ratio is then computed easily:

$\ln [\cdot]:=$ after / before

Out $[\cdot]=0.0202146$ 